\title{
Artrite Reumatoide e a Poluição do Ar
}

\section{Rheumatoid Arthritis and Air Pollution}

Dayane dos Santos Souza Magalhães ${ }^{\mathrm{a}}$; Leticia Rosa Espírito Santo de Freitas ${ }^{\mathrm{a}}$; Maria Amélia dos Santos Peres ${ }^{\mathrm{a}}$; Vander Fernandes $^{\mathrm{a}}$; Walkiria Shimoya-Bittencourt ${ }^{\mathrm{a}}$; Cristhiane Almeida Leite da Silva ${ }^{\mathrm{a}}$

aUniversidade de Cuiabá, Programa de Pós-Graduação Stricto Sensu em Ambiente e Saúde. MT, Brasil.

*E-mail: dayane.souza.enf@gmail.com

\begin{abstract}
Resumo
A artrite reumatoide é uma doença inflamatória, crônica, autoimune, sistêmica e progressiva com etiologia ainda desconhecida, que causa danos progressivos ao sistema musculoesquelético. Esta doença atinge aproximadamente $0,5 \%$ a $1 \%$ da população mundial, predomina no sexo feminino e acomete todas as faixas etárias com maior prevalência entre 40-60 anos. A atividade da doença é avaliada pelo Índice de Atividade da Doença (DAS28) analisando 28 articulações. Estudos demonstram que a poluição atmosférica está associada aos agravos à saúd e humana e à perda de qualidade de vida. Este estudo teve como objetivo relatar o papel da poluição atmosférica na artrite reumatoi de, através de uma revisão narrativa de literatura, a partir das bases de dados PubMed e Scielo. Incluíram-se artigos nos idiomas inglês e português publicados em periódicos, nacionais e internacionais, acerca da temática da pesquisa. A relação entre o desenvolvimento da AR e diferentes poluentes atmosféricos foi analisada em diversos estudos, que demostraram associação entre diferentes poluentes atmosféricos e o desenv olvimento da doença, no entanto, nenhum estudo publicado até a data atual, avaliou a relação com a exacerbação da atividade da doença.
\end{abstract}

Palavras-chave: Artrite Reumatoide. Poluição do Ar. Doenças Autoimunes.

\begin{abstract}
Rheumatoid arthritis is an inflammatory, chronic, autoimmune, systemic and progressive disease with a still unknown etiology that causes progressive damage to the musculoskeletal system. This disease affects approximately $0.5 \%$ to $1 \%$ of the world population, predominates in females and affects all age groups with a higher prevalence between 40-60 years. The disease activity is assessed by the Disease Activity Index (DAS28) analyzing 28 joints. Studies show that air pollution is associated with damage to human health and loss of quality of life. This study aimed to report the role of air pollution in rheumatoid arthritis, through a literature narrative review, using the PubMed and Scielo databases. Articles in English and Portuguese published in national and international journals about the research theme were included. The relationship between the RA development and different air pollutants has been analyzed in several studies, which showed an association between different air pollutants and the development of the disease, however, no study published to date has evaluated the relationship with the disease activity exacerbation.
\end{abstract}

Keywords: Rheumatoid Arthritis. Air Pollution. Autoimmune Diseases.

\section{Introdução}

A artrite reumatoide (AR) é uma doença inflamatória, crônica, autoimune, sistêmica e progressiva com etiologia ainda desconhecida, que causa danos progressivos ao sistema musculoesquelético (MOTA et al., 2011; GOELDNER et al., 2011). Atinge aproximadamente $0,5 \%$ a $1 \%$ da população mundial e predomina de duas a três vezes mais no sexo feminino, todas as faixas etárias com maior prevalência entre 40-60 anos (MOTA et al., 2011; MOTA et al., 2012). No Brasil, acomete até $1 \%$ na população adulta, estimando-se em 1.300.000 o número de pessoas acometidas (MOTA et al., 2011).

A artrite reumatoide afeta grandes e pequenas articulações associado às manifestações sistêmicas como rigidez matinal, fadiga e perda de peso. Quando envolve outros órgãos, a morbidade e a gravidade da doença são maiores, podendo diminuir a expectativa de vida em 3 a 10 anos (MOTA et al., $\mathrm{t}$ al., 2012; ACRS, 2002).
Nos países em desenvolvimento, após dez anos, estima-se que metade desses pacientes sejam incapazes de manter um trabalho de tempo integral (WHO, 2012). Com a progressão da doença, os pacientes desenvolvem incapacidade para realização de suas atividades, tanto de vida diária como profissional, com impacto econômico significativo para o paciente e para a sociedade (ACRS, 2002). Além dos problemas relacionados, os pacientes com a doença também são acometidos por problemas psicológicos, como: ansiedade e depressão com uma prevalência de $13 \%$ a $47 \%$, sendo que esses transtornos são três vezes mais prevalentes nestes pacientes (MELLA et al., 2010).

Pacientes com doenças reumáticas, muitas vezes, se queixam que certas condições meteorológicas e influências climáticas podem agravar o quadro clínico da doença, sendo a artrite reumatoide a doença mais relacionada com essas condições (GORIN et al., 1999; SMEDSLUND et al., 2009). Sendo assim, o objetivo deste estudo foi revisar e descrever o 
papel da poluição atmosférica sobre a artrite reumatoide.

\section{Desenvolvimento}

\subsection{Metodologia}

Realizou-se uma revisão narrativa de literatura com busca nas bases bibliográficas Pubmed e Lilacs, utilizando unitermos em inglês e português. Os descritores utilizados foram: Arthritis, Rheumatoid OR Rheumatoid arthritis AND Air Pollutants OR Air Pollutants OR Carbon Monoxide OR Carbon Monoxide OR Carbon Dioxide OR Carbon Dioxide OR Particulate Matter OR Ozone OR Vehicle Emissions OR Gaseous Pollutants OR Pesticides OR Silicon Dioxide OR Industrial Pollutants. A busca ocorreu no mês de julho de 2020 .

Incluíram-se artigos nos idiomas inglês e português publicados em periódicos, nacionais e internacionais, acerca da temática da pesquisa, sem restrição do ano de publicação. Excluíram-se os artigos, que associavam outros tipos doenças autoimunes ou aqueles em que não foi possível a leitura do artigo na íntegra. Capítulos de livros, manuais, dissertações e teses com a mesma temática também foram considerados.

\subsection{Poluição do ar e a saúde humana}

A poluição corresponde ao acúmulo ambiental de resíduos sólidos, líquidos ou gasosos decorrentes da atividade do homem, sendo a poluição atmosférica a presença desses resíduos gasosos, ou seja, tudo que pode ser vaporizado ou transformado em pequenas partículas, de modo que possa flutuar no ar, deve ser classificado como poluente potencial. Quando existe uma alteração na composição ou nas propriedades do ar atmosférico causadas pela ação do homem, e que gerem a emissão de poluentes em concentrações nocivas e inconvenientes à saúde e ao meio ambiente, diz-se que o ar está poluído (BRITO, 2005). O ar poluído é uma mistura de partículas — material particulado $(\mathrm{PM})$ - e gases que são emitidos para a atmosfera, principalmente por indústrias, veículos automotivos, termoelétricas, queima de biomassa e de combustíveis fósseis.

A rápida urbanização, verificada em todo o Planeta, trouxe um grande aumento no consumo de energia e, também, de emissões de poluentes provenientes da queima de combustíveis fósseis por fontes fixas, como as indústrias, e por fontes móveis, como os veículos automotores. Atualmente, aproximadamente $50 \%$ da população do Planeta vive em cidades e aglomerados urbanos e está exposta a níveis progressivamente maiores de poluentes do ar (SALVI; BARNES, 2009).

Os poluentes podem ser classificados em primários (emitidos diretamente para a atmosfera) e secundários (resultantes de reações químicas entre os poluentes primários) (ARBEX et al., 2012) e podem estar em concentrações, muitas vezes, elevada e acarretar prejuízos à saúde do ser humano (KUNZLI et al., 2010; WHO, 2005).

Estudos demostram que a poluição atmosférica está associada aos agravos à saúde humana e à perda de qualidade de vida (KELLY; FUSSELL, 2012) e que a poluição do ar é capaz de estimular, diretamente, uma resposta inflamatória sistêmica e representar um importante fator de risco para o desenvolvimento de doenças autoimunes (SUN et al., 2016; BERNATSKY et al., 2011 e RITZ, 2010) como a artrite reumatoide (HART, LADEN; PUETT, 2009).

Entre as faixas etárias mais atingidas, pelos efeitos da poluição do ar, estão as crianças e os idosos. Pessoas que já sofrem de problemas respiratórios também se tornam mais suscetíveis a sofrer com a elevação nos níveis de poluentes atmosféricos (MARTINS et al., 2001; SILVA et al., 2013).

Diversos mecanismos têm sido sugeridos para explicar os efeitos adversos dos poluentes aéreos. A explicação mais consistente e mais aceita é a de que altas concentrações de oxidantes e pró-oxidantes contidos nos poluentes ambientais, como material particulado de diversos tamanhos e composição, e nos gases, como $\mathrm{O}_{3}$ e óxidos de nitrogênio, em contato com o epitélio respiratório, provocam a formação de radicais livres de oxigênio e de nitrogênio que, por sua vez, induzem o estresse oxidativo nas vias aéreas. Em outras palavras, um aumento da presença de radicais livres, que não foram neutralizados pelas defesas antioxidantes, inicia uma resposta inflamatória com a liberação de células e mediadores inflamatórios (citocinas, quimiocinas e moléculas de adesão), que atingem a circulação sistêmica, levando a uma inflamação subclínica com repercussão não somente no sistema respiratório, mas também causando efeitos sistêmicos (KUNZLI et al., 2010; WHO, 2005).

Os efeitos dos poluentes sobre a saúde podem ser agudos ou crônicos. Os efeitos agudos se manifestam após um curto espaço de tempo entre a exposição e os efeitos (horas ou dias). Os efeitos crônicos são avaliados, geralmente, em estudos longitudinais com duração de anos ou décadas (BRAGA et al., 2001). Estudos mostram a associação entre poluição do ar e incidência de doenças respiratórias, cardiovasculares, neurológicas e de diversos tipos de câncer (KUNZLI et al., 2010; KAMPA, 2007).

Para o Brasil, a OMS estima que a poluição atmosférica cause cerca de 20 mil óbitos/ano, valor cinco vezes superior ao número de óbitos estimado pelo tabagismo ambiental/ passivo, e 10,7 mil óbitos/ano decorrentes da poluição do ar em ambientes internos (WHO, 2009). A OMS destaca, ainda, que a poluição atmosférica foi a causa de 223 mil mortes por câncer de pulmão em todo o Planeta no ano de 2010 (OMS, 2013).

Solomon et al. (2011) mostram que a diminuição de 10 $\mathrm{mg} / \mathrm{m} 3$ do material particulado presente na atmosfera com até $2,5 \mu m$ representaria um aumento em 1,5 para a expectativa de vida das pessoas.

\subsection{A poluição do ar e a Artrite Reumatoide}

A etiologia das doenças reumáticas ainda é um assunto 
bastante controverso e diversos estudos apontam o papel fundamental da interação entre os fatores genéticos e os fatores ambientais. Gawda et al. (2017) sugerem, ainda, que o impacto dos fatores ambientais não pode ser subestimado e que outros fatores como: agentes infecciosos, medicamentos, agentes físicos, tais como a exposição ao frio ou a poluição do ar, são considerados como fatores de risco para o desenvolvimento ou exacerbação destas condições.

A poluição do ar é apontada, em alguns estudos, como um fator de risco plausível para o desenvolvimento de doenças autoimunes (SUGIYAMA et al., 2010; STOLT et al., 2005; BERMATSKY, et al., 2016), estando associada a doenças reumáticas, entre essas Lúpus Eritematoso Sistêmico (BERNATSKY, 2011; ALVES et al., 2018) Artrite Reumatoide (HART et al., 2009; HART et al., 2013; HART et al., 2013b; CHANG et al., 2016; DE ROSS et al., 2014; JUNG et al., 2017), Artrite Idiopática Juvenil (SUN et al., 2016; ZEFT et al., 2010) e outras.

Uma relação entre a exposição a partículas no ar e elevações nos níveis de marcadores inflamatórios foram descritos (TORNQYIST et al., 2007; SALIN et al., 2014; MEIER, et al., 2014; ZHAO et al., 2013; BIND et al., 2012; HAJAT et al., 2015). Estudos demonstram que a poluição do ar é capaz de estimular, diretamente, uma resposta inflamatória sistêmica (SALIM et al., 2014), bem como específica dos tecidos (KUNZLI; PEREZ; RAPP, 2010), sendo que o material particulado inalado pode induzir inflamação pulmonar local, bem como inflamação sistêmica (KUNZLI et al., 2005; LADEN et al., 2006; NAFSTAD et al., 2003; PENAD-MORAND et al., 2005; PEREIRA et al., 2005; POPE et al., 2002; POPE; DOCKERY, 2006; SUNYER, 2001; VAN EEDEN, 2005).

Muitos dos efeitos nocivos para a saúde humana causada por poluentes atmosféricos têm sido associados às partículas finas e ultrafinas. Estas partículas podem ser transferidas para o sistema sanguíneo e desencadear uma resposta inflamatória sistêmica e a exacerbação de muitas patologias, como as doenças sistêmicas autoimunes (FARHAT, 2011).

A exposição à poluição do ar gera estresse oxidativo nas vias aéreas, fazendo com que células epiteliais das vias aéreas e macrófagos alveolares expressem citocinas próinflamatórias, que desencadeiam a maturação de células dendríticas residentes nas vias aéreas, que por sua vez migram para os gânglios linfáticos. Em um indivíduo em que os linfócitos autorreativos escaparam aos mecanismos de tolerância central na medula óssea e timo, essas células dendríticas ativadas apresentam autoantígenos, no contexto de moléculas coestimulatórias, ativando os linfócitos, que em vez de tolerá-los, desencadeiam o desenvolvimento de uma resposta autoimune e uma vez que tal resposta autoimune é estabelecida (mesmo nos casos em que exposição à poluição não foi um fator nas etapas de iniciação), as citocinas próinflamatórias geradas pela exposição à poluição do ar irão atuar para consolidar e exacerbar a resposta inflamatória
(RITZ, 2010).

Estudos em pacientes com artrite reumatoide, demonstram que em particular, espécies reativas livres de oxigênio liberadas após a inalação de MP finos e ultrafinos ou poluentes gasosos inalados no trato respiratório são capazes de ativar o $\mathrm{NF}-\mathrm{kB}$, um regulador chave para o controle pró-inflamatório e a produção de citocinas (FARHAT, 2011; YING et al., 2015; RITZ, 2010) ativando linfócitos $\mathrm{T}$ helper tipo 1 (Th1), levando produção de fator de necrose tumoral alfa, interleucina-1 e interleucina-6 (YING et al., 2015). Estas citocinas estimulam monócitos em repouso a se diferenciarem em células dendríticas que então apresentam autoantígenos, coestimulando linfócitos $\mathrm{T}$ autorreativos, que migram para tecidos-alvo (preferencialmente, articulações sinoviais), causando destruição de células (RITZ, 2010).

Existem poucos estudos, que avaliaram a associação entre a exposição à poluição do ar e o início ou agudizações de doenças autoimunes em crianças e adolescentes. Zeft et al. (2009) mostraram um maior risco de início de sintomas de artrite reumatoide juvenil em crianças menores de cinco anos de idade, associado com maiores concentrações de $\mathrm{PM}_{2,5} \mathrm{e}$ condições do ar estagnado nos últimos 14 dias $(\mathrm{RR}=1,60$, $95 \%$ IC 1,00 - 2,54).

Hart et al. (2009), através de uma coorte prospectiva, examinaram a associação entre a distância de uma estrada e a incidência de artrite reumatoide. Foram avaliadas 90.297 mulheres americanas no Nurses Health Study (NHS), sugerindo que uma maior exposição à poluição do tráfego pode estar associada ao risco de desenvolver artrite reumatoide, sendo este um novo fator de risco ambiental para a doença pesquisada na população adulta.

Hart et al. (2012), em um Estudo Epidemiológico Sueco de Artrite Reumatoide (EIRA), analisaram se a exposição a longo prazo à poluição do ar estava associada ao risco de artrite reumatoide. 1.497 casos de $\mathrm{AR}$ incidentes e 2.536 controles e os níveis de $\mathrm{PM}_{10}$ e os gases poluentes $\left(\mathrm{SO}_{2}\right.$ e $\left.\mathrm{NO}_{2}\right)$ do tráfego e do aquecimento interno de casas previsto para todos os endereços residenciais foram analisados. Não houve evidência de aumento do risco de $\mathrm{AR}$ com exposição ao $\mathrm{PM}_{10}$. Os riscos totais de artrite reumatoide foram elevados para os poluentes gasosos, mas não foram estatisticamente significativos após o ajuste para tabagismo e educação (OR = 1,18 [IC de 95\%: 0,97-1,43] e OR = 1,09 [IC 95\%: 0,99-1,19] para SO2 e NO2 no $10^{\circ}$ ano antes do início). Este estudo concluiu que não há associações globais consistentes entre a poluição do ar na área de Estocolmo e risco para artrite reumatoide.

Em um estudo de caso-controle, conduzido na Suécia, Hart et al. (2013b) avaliaram 111.425 participantes do NHS com informações sobre exposição à poluição atmosférica, bem como dados relativos a outras exposições ao estilo de vida e desfechos da doença. Níveis externos de diferentes frações de tamanho de material particulado $\left(\mathrm{PM}_{10} \mathrm{ePM}_{2.5}\right) \mathrm{e}$ poluentes gasosos $\left(\mathrm{SO}_{2}\right.$ e $\left.\mathrm{NO}_{2}\right)$ foram previstos para todos os endereços. O estudo encontrou pequenos aumentos no risco 
de artrite reumatoide associado com exposição ao $\mathrm{SO}_{2}$ nos $10^{\circ}$ e $20^{\circ}$ anos antes do início da doença e uma associação com $\mathrm{NO}_{2}$ apenas para casos negativos para auto anticorpos. $\mathrm{O}$ mesmo grupo não encontrou associação com $\operatorname{PM}_{10}(\leq 10 \mu \mathrm{m}$ em diâmetro aerodinâmico).

Chang et al. (2016) conduziram um estudo retrospectivo, utilizando dados coletados do Banco de Dados de Seguro de Saúde Longitudinal (LHID), mantido pela Secretaria de Seguro Nacional de Saúde de Taiwan e o Banco de Dados de Monitoramento da Qualidade do Ar de Taiwan (TAQMD. Uma área residencial foi definida de acordo com a localização da clínica e do hospital, que tratavam de infecções agudas do trato respiratório superior. As concentrações médias anuais de poluentes do ar foram categorizadas em 4 níveis baseados em quartis. Foi avaliado o risco de artrite reumatoide em residentes expostos a 4 níveis de concentrações de $\mathrm{PM}_{2.5} \mathrm{e}$ $\mathrm{NO}_{2}$. Este estudo possibilitou detectar um aumento do risco de AR em participantes expostos a $\mathrm{PM}_{2,5}$ e $\mathrm{NO}_{2}$. Entre os quatro quartis de concentração de $\mathrm{NO}_{2}$, ou seja, Q1, Q2, Q3 e Q4, as razões de risco ajustadas (aHRs) em Q2, Q3 e Q4 comparadas com isso no Q1 foram 1,07 (IC de 95\% = 0,761,50), 1,63 (IC 95\% = 1,16-2,31), e 1,49(IC95\% = 1,05-2,12), respectivamente. Em relação às concentrações de PM2,5, os aHRs após a exposição ao Q2, Q3, e os níveis de Q4 foram $1,22(\mathrm{IC} 95 \%=0,85-1,74), 1,15$ (IC 95\% = 0,82-1,62) e 0,79 (IC $95 \%=0,53-1,16)$, respectivamente. Os resultados deste estudo nacional sugerem um aumento do risco de desenvolver artrite reumatoide em residentes expostos ao $\mathrm{NO}_{2}$.

De Ross et al. (2014), em seu estudo, demonstraram maior risco de desenvolver AR com a vida perto do trânsito; no entanto, há evidências sugerindo que a poluição do ar pode não ser responsável por essa associação. O ruído, outra exposição gerada pelo tráfego, não foi estudado como um fator de risco para a AR. O estudo investigou a proximidade do tráfego, poluição do ar ambiente e ruído da comunidade em relação à doença nas regiões de Vancouver e Victoria, na Colúmbia Britânica, Canadá. A incidência de AR foi aumentada com a proximidade do tráfego, com um OR de 1,37(IC 95\%: 1,11, $1,68)$ para residência $\leq 50 \mathrm{~m}$ de uma estrada em comparação com a residência $>150 \mathrm{~m}$ de distância. Não foi encontrada associação com exposições relacionadas ao tráfego, como $\mathrm{PM}_{2.5}$, óxidos de nitrogênio ou ruído. O ozônio no nível do solo, que era mais alto nas áreas suburbanas, foi associado a um risco aumentado de artrite reumatoide $(\mathrm{OR}=1,26$; IC 95\%: 1,18, 1,36). O estudo conclui que há uma associação previamente observada de risco de artrite reumatoide com proximidade do tráfego e sugere que nem os níveis de ruído nem os poluentes atmosféricos relacionados com o tráfego são responsáveis por este problema.

Um estudo de Jung et al. (2017), uma coorte retrospectiva, incluiu 322.301 indivíduos entre 30 e 50 anos, selecionados da Base Nacional de dados de pesquisa de seguro de saúde em Taiwan, que foram seguidos de 2001 a 2010. Foi utilizado um modelo de Cox estendido e dependente do tempo para estimar as associações entre as concentrações médias anuais de poluentes atmosféricos com $\mathrm{AR}$, incluindo $\mathrm{CO}, \mathrm{NO}_{2}$ $\mathrm{O}_{3}$, partículas com diâmetro aerodinâmico inferior a $10 \mu \mathrm{m}$ $\left(\mathrm{PM}_{10}\right)$, e $\mathrm{SO}_{2}$, e relataram a taxa de risco OR e IC $95 \%$. Foi encontrada artrite reumatoide recém-diagnosticada associada positivamente com aumento $100 \mathrm{ppb}$ de $\mathrm{CO}$ (OR ajustado $=1,17$ [IC 95\% = 1,16, 1,18]), um aumento de $10 \mathrm{ppb}$ aumento em $\mathrm{NO}_{2}(1,54[1,45 ; 1,64])$, um aumento de $10 \mathrm{ppb}$ no $\mathrm{O}_{3}(1,37[1,33,1,41])$ e 1 ppb em $\mathrm{SO}_{2}(1,02[1,00,1,04])$. Não houve associação entre $10-\mu \mathrm{g} / \mathrm{m}^{3}$ aumento em PM e artrite reumatoide $(1,02[0,99,1,05])$. Os achados do estudo sugerem que o $\mathrm{O}_{3}$ e poluentes atmosféricos relacionados ao tráfego $\left(\mathrm{CO}\right.$ e $\left.\mathrm{NO}_{2}\right)$ estão, positivamente, associados com a maior incidência de artrite reumatoide.

Segundo Gorin et al. (2009), pacientes com doenças reumáticas, muitas vezes, se queixam que certas condições meteorológicas podem agravar o quadro clínico da doença. Outros estudos sobre influência de condições climáticas cita a artrite reumatoide como a doença mais relacionada com essas condições (VAN EEDEN, 2005).

\section{Conclusão}

Através dos estudos encontrados e analisados se pode demostrar que há associação entre o desenvolvimento da artrite reumatoide e a poluição do ar, porém não foi identificado nenhum estudo publicado que avaliou a relação entre a exacerbação da doença e a poluição do ar.

\section{Referências}

ARBEX, M.A.B.D.O. et al. Poluição do ar e o sistema respiratório. J. Bras. Pneumol., v. 38, n.5, p. 643-655, 2012. doi: 10.1590/S1806-37132012000500015

ALVES, A.G.D, et al. Influence of air pollution on airway inflammation and disease activity in childhood-systemic lupus erythematosus. Clin. Rheumatol., v. 37, p. 683-690, 2018. doi: 10.1007/s10067-017-3893-1

\section{AMERICAN COLLEGE OF RHEUMATOLOGY} SUBCOMMITTE on Rheumatoid Arthritis Guidelines. Guidelines for the management of rheumatoid arthritis. Arthritis Rheum, v.46, p.328-346, 2002.

BERNATSKY, S. et al. Associations between ambient fine particulate levels and disease activity in patients with Systemic Lupus Erythematosus (SLE). Environ Health Perspect., v. 119, p. 45-49. 2011. doi: 10.1289/ehp.1002123

BERNATSKY, S. et al. "Fine particulate air pollution and systemic autoimmune rheumatic disease in two Canadian provinces," Environmental Research., v. 146, p. 85-91, 2016. doi: 10.1016/j.envres.2015.12.021

BIND, A.A. et al. Air pollution and markers of coagulation, inflammation, and endothelial function: associations and epigeneenvironment interactions in an elderly cohort," Epidemiology, v.23, n. 2, p. 332-340, 2012. doi: 10.1097/EDE.0b013e31824523f0

BRAGA, A.L.; ZANOBETTI, A.; SCHWARTZ, J. The lag structure between particulate air pollution and respiratory and cardiovascular deaths in 10 US cities. J Occup Environ Med., v. 43, n. 11, p. 927-933. doi: 10.1097/00043764-200111000-00001

BRITO, H.P. Análise das emissões atmosféricas por veículos 
automotores em Natal-RN, 2005, 123f. Dissertação (Mestrado em Engenharia Mecânica). Universidade Federal do Rio Grande do Norte, 2005.

CHANG K.H; H.S.U. et al. Air pollution exposure increases the risk of rheumatoid arthritis: a longitudinal and nationwide study. Environment International, v. 94, p. 495- 499, 2016. doi: 10.1016/j.envint.2016.06.008

DE ROOS, A.J. et al. Proximity to traffic, ambient air pollution, and community noise in relation to incidente rheumatoid arthritis. Environ Health Perspect., v. 122, n.10; p.1074-1080, 2014. doi: 10.1289/ehp.1307413

GAWDA, A. et al. Air pollution oxidative stress, and exacerbation of autoimune

diseases. Cent. Eur. J. Immunol., v.42, n. 3, p. 305-312, 2017. doi: 10.5114 / ceji.2017.70975

GOELDNER, I. et al. Artrite reumatoide: uma visão atual. Rev. Bras Patol Med Lab., v. 47, n.5, p. 495-503, 2011. doi: 10.1590/ S1676-24442011000500002

GORIN, A.A. et al. Rheumatoid arthritis patients show weather sensitivity in daily life, but the relationship is not clinically significant. Pain., v., 81, p. 173-177, 1999. doi: 10.1016/S03043959(99)00010-X

HART, J.E. et al. Exposure to traffic pollution and increased risk of rheumatoid arthritis. Environ Health Perspect., v. 117, p. 10651069, 2009. doi: 10.1289 / ehp.0800503

HART, J.E. et al. Ambient air pollution exposures and risk of rheumatoid arthritis: results from the swedish eira case-control study. Ann Rheum Dis., v. 72, n. 6, p. 888-894, 2013. doi:10.1136/ annrheumdis-2012-201587

HAJAT, A. et al. Long-term exposure to air pollution and markers of inflammation, coagulation, and endothelial activation a repeatmeasures analysis in the multi-ethnic study of atherosclerosis (MESA). Epidemiology, v. 26, n. 3, p. 310-320, 2015. doi: 10.1097 / EDE.0000000000000267

JUNG, C.R.; HSIEH, H.Y.; HWANGA, B.F. Air Pollution as a Potential Determinant of Rheumatoid Arthritis: A Populationbased Cohort Study in Taiwan. Epidemiology., v. 28, p. S54-S59, 2017. doi: 10.1097/EDE.0000000000000732

KAMPA, M.; CASTANAS, E. Human health effects of air pollution. Environ Pollut., v. 151, n. 2, p. 362-367, 2008. doi:10.1016/j.envpol.2007.06.012

KELLY, F.J.; FUSSELL, J.C. Size, source and chemical composition as determinants of toxicity attributable to ambient particulate matter. Atmos Environ., v. 60, p. 504-526, 2012. doi:10.1016/j.atmosenv.2012.06.039

KUNZLI, N.; PEREZ, L.; RAPP, R. Air quality and health. Lausanne: European Respiratory Society; 2010.

KÜNZLI, N. et al. Ambient air pollution and atherosclerosis in Los Angeles. Environ Health Perspect., v. 113, p. 201-206, 2005. doi: 10.1289 / ehp. 7523

LADEN, F. et al. Reduction in fine particulate air pollution and mortality: extended follow-up of the Harvard Six Cities study. Am J Respir Crit Care Med., v. 173, n. 6, p. 667-672. 2006. doi: 10.1164 / rccm.200503-443OC

MARTINS, L.C. et al. Relação entre poluição atmosférica e atendimentos por infecção de vias aéreas superiores no município de São Paulo: avaliação do rodízio de veículos. Revista Brasileira de Epidemiologia., v.4, n.3, p.220-229, 2001. doi:10.1590/S1415790X2001000300008

MELLA, L.F.B.; BERTOLO, M.B.; DALGALARRONDO,
P. Depressive symptoms in rheumatoid arthritis patients. Rev Bras Psiquiatr., v. 32, p. 257-263, 2010. doi:10.1590/S151644462010005000021

MEIER, R. et al. Associations of short-term particle and noise exposures with markers of cardiovascular and respiratory health among highway maintenance workers. Environmental Health Perspectives., v.122, n.7, p. 726-732, 2014. doi: 10.1289 / ehp. 1307100

MOTA, L.M.H. et al. Consenso da Sociedade Brasileira de Reumatologia 2011 para o diagnóstico e avaliação inicial da artrite reumatoide. Rev Bras Reumatol., 51, p. 207-219, 2011. doi:10.1590/S0482-50042011000300002

MOTA, L.M.H. et al. Consenso da Sociedade Brasileira de Reumatologia 2012 para o tratamento da artrite reumatoide. Rev. Bras. Reumatol., v. 52, p. 152-174, 2012. doi: 10.1590/S048250042012000200002

NAFSTAD, P. et al. Lung cancer and air pollution: a 27 year follow up of 16,209 Norwegian men. Thorax., v.58, n. 12, p. 1071-1076, 2003. doi: 10.1136 / tórax.58.12.1071

OMS. 2013. "Outdoor Air Pollution a Leading Environmental Cause of Cancer Deaths." Edited by OMS. Geneva. http://www. iarc.fr/en/media-centre/iarcnews/pdf/pr221_E.pdf.

PENARD-MORAND, C. et al.. Long-term exposure to background air pollution related to respiratory and allergic health in schoolchildren. Clin Exp Allergy., v. 35, n.10, 1279-1287, 2005. doi: 10.1111 / j.1365-2222.2005.02336.x șepiPEREIRA,

F.A. et al. Influence of air pollution on the incidence of respiratory tract neoplasm. J. Air Waste Manag Assoc., v.55, n.1, p 83-87, 2005. doi: $10.1080 / 10473289.2005 .10464603$

POPE, C.A. III. et al. Lung cancer, cardiopulmonary mortality, and long-term exposure to fine particulate air pollution. JAMA., v.287, n.9, p.1132-1141, 2002. doi: 10.1001 / jama.287.9.1132

POPE, C.A. III; DOCKERY, D.W. Health effects of fine particulate air pollution: lines that connect. J. Air Waste Manage Assoc., v.56, p.709-742, 2006. doi: 10.1080/10473289.2006.10464485

RITZ, S.A. Air pollution as a potential contributor to the 'epidemic' of autoimmune disease. Med Hypotheses., v.74, p.110-117, 2010. doi: $10.1016 /$ j.mehy.2009.07.033

SALVI, S.S.; BARNES, P.J. Chronic obstructive pulmonary disease in non-smokers. Lancet., v.374, n. 9691, p.733743, 2009. doi:10.1016/S0140-6736(09)61303-9

SALIM, S.Y.; KAPLAN, G.G.; MADSEN, K.L. Air pollution effects on the gut microbiota: a link between exposure and inflammatory disease. Gut Microbes., v.5, n.2, p.215-219, 2014. doi: $10.4161 /$ gmic. 27251

SILVA, C.M.A. et al. Material particulado originário de queimadas e doenças respiratórias. Revista de Saúde Pública., v.7, n.2, p.345-352, 2013. doi:10.1590/S0034-8910.2013047004410

SMEDSLUND, G. et al. Does the weather really matter? A cohort study of influence of weather and solar conditions on daily variations of joint pain in patients with rheumatoid arthritis. Arthritis Rheum., v. 61, n. 9, p.1243-1247, 2009. doi: 10.1002/ art.24729

SOLOMON, P.A. et al. Air Pollution and health: bridging the gap from sources to health outcomes: conference summary. Air Quality, Atmosphere \& Health, v.5, 2011. doi: 10.1007/s11869011-0161-4.

SUGIYAMA, D.K. et al. Impact of smokin as a risk factor for developing rheumatoid arthritis: a metaanalysis of observational studies. Ann. Rheumatic Dis., v.69, n.1, p.70-81, 2010. doi: 10.1136/ard.2008.096487 
SUNYER, J. Urban air pollution and chronic obstructive pulmonary disease: a review. Eur Respir J., v.17, n.5, p.10241033, 2001. doi: 10.1183 / 09031936.01 .17510240

SUN, G. et al. Association between Air pollution and the development of rheumatic disease: a systematic review. Int. J. Rheumatol., v.25, 2016. doi: 10.1155/2016/5356307

STOLT, P. et al. Silica exposure is associated with increased risk of developing rheumatoid arthritis: Results from the Swedish EIRA Study, Ann. Rheum. Dis., v. 64, n. 4, p. 582-586, 2005. doi: $10.1136 /$ ard.2004.022053

TORNQVIST, H. et al. Persistent endothelial dysfunction in humans after diesel exhaust inhalation. Am. J. Respiratory Crit. Care Med., v.176, n.4, p.395-400, 2007. doi: 10.1164 / rccm.200606-872OC

VAN EEDEN, S.F. et al. Systemic response to ambient particulate matter: relevance to chronic obstructive pulmonary disease. Proc. Am. Thorac. Soc., v.2, n.1, p.61-67. doi: 10.1513 / pats.200406035MS

YING, G. et al. Lipid peroxidation mediated inflammation promotes cell apoptosis through activation of NF- $\kappa \mathrm{B}$ pathway in rheumatoid arthritis synovial cells. Mediators Inflamm., v.5, 2015. doi: 10.1155 / 2015/460310

WHO - World Health Organization. Chronic rheumatic conditions. Disponível em: http://www.who.int/chp/topics/ rheumatic/en/.2012.

WHO - World Health Organization. Air Quality Guidelines Global Update 2005: Report on a Working Group Meeting. WHO Regional Office for Europe, p. 1-30, 2005. http://www.euro.who. int/_data/assets/pdf_file/0008/147851/E87950.pdf

WHO - World Health OrganizatioN. Global health risks. Mortality and burden of disease attributable to selected major risks. Geneva: World Health Organization; 2009.

ZEFT, A.S. et al., Juvenile idiopathic arthritis and exposure to fine particulate air pollution. Clinical and Experimental Rheumatology., v. 27, n. 5, p. 877-884, 2009. PMID: 19917177.

ZHAO, J. et al. The biological effects of individual-level PM2.5 exposure on systemic immunity and inflammatory response in traffic policemen. Occup. Environ. Med., v. 70, n. 6, p. 426-431, 2013. doi: 10.1136 / oemed-2012-100864 . 